\title{
Implementação de um módulo IoT para controle de luminárias LED baseado em espelho de corrente com chaveamento de ramos
}

\author{
Rubens A. Fernandes *. Marcus V. M. Melo** \\ Lennon B. F. Nascimento***. Karolayne B. Martins****. Israel G. Torné *****. Raimundo C. S. Gomes****** \\ *HUB: Tecnologia e Inovação, Universidade do Estado do Amazonas, Manaus, AM \\ Brasil (Tel: +5592 98212-9068; e-mail: rdafr.eng@uea.edu.br). \\ **HUB: Tecnologia e Inovação, Universidade do Estado do Amazonas, Manaus, AM \\ Brasil (Tel: +5592 98184-4946; e-mail: mvmm.eng17@uea.edu.br). \\ *** HUB: Tecnologia e Inovação, Universidade do Estado do Amazonas, Manaus, AM \\ Brasil (Tel: +55 92 99158-8482; e-mail: lbfn.eng@uea.edu.br). \\ **** HUB: Tecnologia e Inovação, Universidade do Estado do Amazonas, Manaus, AM \\ Brasil (Tel: +5592 99324-7695; e-mail: kbm.ele16@uea.edu.br). \\ *****HUB: Tecnologia e Inovação, Universidade do Estado do Amazonas, Manaus, AM \\ Brasil (Tel: +5592 98137-9433; e-mail: igondrest@gmail.com). \\ ******HUB: Tecnologia e Inovação, Universidade do Estado do Amazonas, Manaus, AM \\ Brasil (Tel: +55 92 98816-0888; e-mail: rsgomes@uea.edu.br).
}

\begin{abstract}
The paradigm of Internet of Things has assumed prominence for specific and global solutions in the most varied systems, having as main element the information flowing between the different processes. One area susceptible to the Internet of Things is Lighting. Today there is a shortage of solution in Smart Lamps. Therefore, the purpose of this article is to propose a solution that integrates the concept of Internet of Things and lighting converging into smart lamps. This solution will use concepts related to transistor circuits responsible for current control in LED luminaires as well as the User Datagram Protocol and Transmission Control Protocol that are responsible for the system communication rules. The implementation of this module will be able to make LED luminaires, which are currently passive elements in buildings, into luminaires that offer the user remote lighting control possibilities.
\end{abstract}

Resumo: O paradigma de comunicação Internet das Coisas assumiu destaque para soluções específicas e globais nos mais variados sistemas, tendo como elemento principal a informação fluindo entre os diferentes processos. Uma área susceptível à aplicação de Internet das Coisas é a iluminação. Atualmente existe uma carência de soluções em luminárias inteligentes. Por isso, o objetivo deste artigo é propor uma solução que integre o conceito de Internet das Coisas e iluminação para a convergência em iluminação inteligente. Essa solução utilizará de conceitos relacionados a circuitos transistorizados responsáveis pelo controle de corrente em luminárias LEDs assim como o User Datagram Protocol e Transmission Control Protocol que são responsáveis pelas regras de comunicação do sistema. A implementação desse módulo será capaz de tornar as luminárias LEDs, que atualmente são elementos passivos em edifícios, em luminárias que disponibilizam ao usuário possibilidades de controle de iluminação de forma remota.

Keywords: LED luminaires; smart lighting; wireless communication systems; Internet of Things; current mirror; microelectronics.

Palavras-chaves: Luminárias Led; iluminação inteligente; sistemas de comunicação sem fio; internet das coisas; Espelho de Corrente; microeletrônica.

\section{INTRODUÇÃO}

Grande parte dos dispositivos eletroeletrônicos pré-existentes não possuem elementos que lhe possibilitem comunicação remota. Mais da metade deles não compartilham os dados entre si ou com as tecnologias de armazenamento em nuvem (Ala Al-Fuqaha 2015). A tecnologia que favoreceria a intercomunicação entre esses sistemas seria a Internet das Coisas, largamente conhecida como Internet of Things (IoT).
A Internet das coisas (IoT) é um paradigma de comunicação em evidência atualmente. Ela adquiriu relevância na indústria e na comunidade acadêmica por ser um dos pilares para quarta revolução industrial, também denominada no Brasil como Indústria 4.0 (C. Perera et al 2014). Esse paradigma consiste na conexão de objetos comuns à internet. Esses objetos são dotados de transceptores para comunicação digital e pilhas de protocolos adequados que possibilitarão uma intercomunicação entre eles. A partir dessa perspectiva de comunicação entre dispositivos, a IoT dará a oportunidade de 
desenvolvimento de aplicações com diferentes finalidades que utilizem a quantidade significativa de dados geradas por esses dispositivos para assim disponibilizar aos usuários novos serviços (J.ChandraMohan 2017).

Aplicações de IoT são encontradas tanto na literatura e teoria quanto na parte de desenvolvimento prático. Atualmente já são observados no âmbito de transporte, veículos, agricultura, saúde, mercados e indústrias (A. A.-Fuqaha et al. 2015). Elas possuem suas particularidades de acordo com as áreas que estão inseridas. No caso da iluminação, existe o conceito da iluminação inteligente ou smart lighting. Existem grandes desafios para IoT nessa área, haja visto o número limitado de tecnologias desenvolvidas. Além disso, o custo das soluções existentes se torna uma barreira para transição de uma iluminação passiva para iluminação controlável e comunicável. Tendo isso em vista, será abordado neste artigo a implementação de um módulo IoT que fornecerá recursos para as luminárias LED (Light Emissor Diode) comuns atualmente instaladas nas plantas de iluminação.

\section{MATERIAIS E MÉTODOS}

\subsection{Diodo Emissor de Luz}

O Diodo Emissor de Luz ou LED é a tecnologia de fonte luminosa mais eficiente disponível atualmente, devido a sua eficácia e vida útil duradoura. Algumas de suas características são a alta corrente de operação e o fluxo luminoso que depende da corrente aplicada, a qual deve ser adequadamente regulada para manter a intensidade luminosa desejada.

Para representar a String de LEDs, foi utilizada uma luminária pública contendo um Chip on board (CoB) de LEDs de alto brilho com potência de $50 \mathrm{~W}$, que pode emitir até $5000 \mathrm{~lm}$ de fluxo luminoso e possui eficiência luminosa de aproximadamente $90 \mathrm{~lm} / \mathrm{W}$.

\subsection{Transistores}

Como o sistema de LEDs utilizado possui corrente nominal elevada e os transistores utilizados em espelhos de corrente operam em regiões lineares, os transistores bipolares utilizados no dispositivo devem possuir encapsulamento robusto e capacidade de condução de corrente considerável. Além disso, visando diminuir o erro gerado pela razão de transferência, ou seja, o valor da razão entre a corrente de coletor e a corrente de base do semicondutor escolhido deve ser suficientemente grande para minimizar este fator.

A chave eletrônica utilizada foi um transistor de efeito de campo de semicondutor de óxido metálico, ou do inglês MOSFET, que receberia então o sinal do GPIO, do inglês General Purpose Input/Output, para entrada do ramo ao sistema. O MOSFET escolhido também deve possuir baixa resistência entre dreno e fonte quando acionado $\left(\mathrm{R}_{\mathrm{ds}(\mathrm{on})}\right)$, para reduzir as perdas de condução na corrente de base dos transistores bipolares.

Considerando estas características, o transistor bipolar escolhido foi o BD135, que possui corrente nominal de coletor de até 1,5 A, com $\beta$ de aproximadamente 150 para temperaturas de $25^{\circ} \mathrm{C}$, suportando tensões entre coletor e emissor de até $45 \mathrm{~V}$, o que o torna adequado para utilização em conjunto com o $\mathrm{CoB}$ escolhido. Vale ressaltar que $\beta$ é a relação entre a corrente de coletor e a corrente de base, denominado ganho estático de corrente. O MOSFET utilizado foi o IRF3205, que possui $\mathrm{R}_{\mathrm{ds}(\text { on) }}$ de $8 \mathrm{~m} \Omega$.

\subsection{Espelho de corrente}

O uso de espelhos de corrente é uma alternativa para homogeneizar a corrente nos aglomerados de LEDs, a fim de garantir que a corrente que circula em cada ponto de LED seja um reflexo da corrente de referência. $\mathrm{O}$ espelho de corrente evitará que o brilho total seja reduzido e que o brilho de cada string de LED se torne desigual (J. Kim and S. Park 2016). Como a corrente em todos os pontos será igual à corrente de referência, todos os LEDs apresentarão a mesma intensidade luminosa. Para assegurar que os espelhos de corrente funcionem conforme o esperado é essencial que os transistores utilizados possuam as mesmas características construtivas. A figura 1 é um exemplo de circuito que utiliza espelho de corrente com múltiplos ramos para acender LEDs.

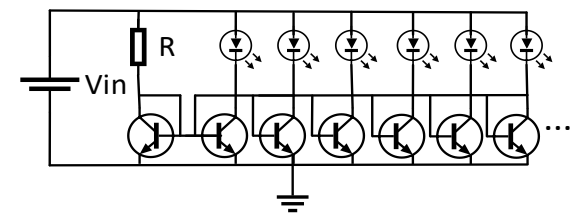

Figura 1 - Circuito com espelho de corrente para acionamento de LEDs.

Os espelhos de correntes são circuitos onde dois transistores são combinados na polarização base-emissor, para garantir que uma corrente constante gerada no primeiro transistor, seja espelhada no segundo, a fim de que o esforço para gerar uma corrente estável não seja repetido em cada estágio do circuito. Isso ocorre quando uma corrente de referência $\mathrm{I}_{\text {ref }}$ é aplicada no transistor $\mathrm{Q}_{1}$, que está ligado como diodo, estabelecendo uma tensão $\mathrm{V}_{\text {be }}$ para ambos os transistores, e um mesmo valor de corrente de emissor. Assim, esse valor de tensão de baseemissor, forçará uma corrente no transistor $\mathrm{Q}_{2}$. Desprezando a corrente de base, para um valor de $\beta$ bem elevado, pode-se garantir que a mesma corrente que passa por $\mathrm{Q}_{1}$ está sendo espelhada em $\mathrm{Q}_{2}$ (SEDRA, S. 2005). Essa propriedade pode ser visualizada na figura 2 a seguir:

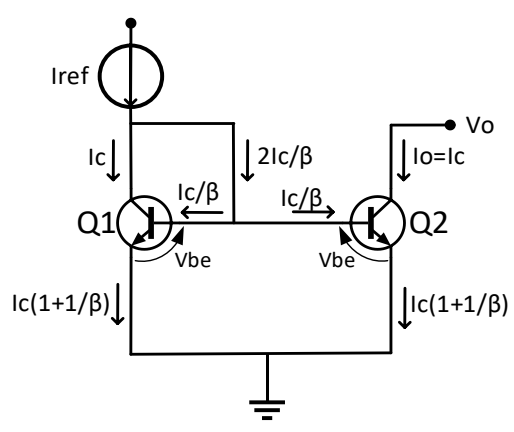

Figura 2 - Espelho de corrente. 
Uma limitação desse tipo de espelho é que, para valores finitos de $\beta$, ou $h_{\mathrm{FE}}$, uma pequena corrente circula pela base dos transistores, o que causa um erro na intensidade da corrente espelhada. Nesse caso, a corrente no coletor de $\mathrm{Q}_{1}$ é calculada a partir da equação 1 :

$$
\text { Ic }=\text { Iref }-\frac{2 \mathrm{Ic}}{\beta}
$$

$\mathrm{I}_{\mathrm{C}}$ é a corrente que passa no coletor de $\mathrm{Q}_{1}$. A partir desta equação, obtemos a equação 2 , isolando $\mathrm{I}_{\text {ref: }}$ :

$$
\text { Iref }=\text { Ic }+\frac{2 \text { Ic }}{\beta}=\operatorname{Ic}\left(1+\frac{2}{\beta}\right)
$$

Dado que a corrente $\mathrm{I}_{\mathrm{O}}$, que passa no coletor de $\mathrm{Q}_{2}$, é igual a corrente $\mathrm{I}_{\mathrm{C}}$ que passa no coletor de $\mathrm{Q}_{1}$, obtém-se a razão de transferência de corrente:

$$
\frac{\text { Io }}{\text { Iref }}=\frac{\text { Ic }}{\text { Ic }\left(1+\frac{2}{\beta}\right)}=\frac{1}{1+\frac{2}{\beta}}
$$

Para valores finitos de $\beta$ é gerado um erro que aumenta à medida que a razão de transferência de corrente cresce.

\subsection{Arquitetura de hardware}

O uso de um circuito para controle de iluminação aplicado ao contexto de Internet das Coisas necessita de elementos de comunicação que possibilitem a interoperabilidade desse dispositivo com o usuário final. A figura 3 apresenta um diagrama em blocos que representa a arquitetura de hardware do sistema implementado.

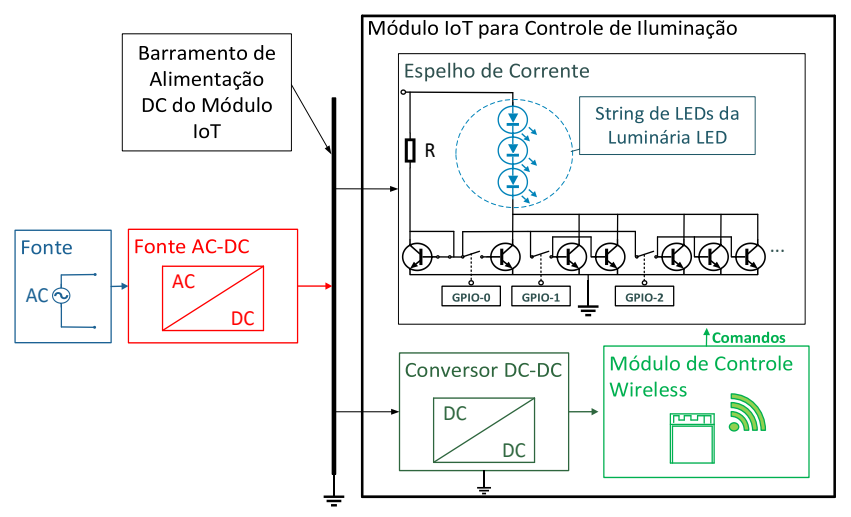

Figura 3 - Visão geral da arquitetura de hardware do sistema.

O modelo apresentado na figura 3 apresenta as seguintes características:

- A corrente espelhada em cada transistor é regulada através do resistor $\mathrm{R}$, no ramo principal;

- O controle de iluminação é realizado mediante o acionamento individual de cada ramo do espelho de corrente projetado;
- Um ramo do espelho de corrente pode conter um ou mais transistores bipolares, permitindo que cada acionamento possa levar a diferentes níveis de condução de corrente;

- Cada ramo do sistema é acionado individualmente por um GPIO de um módulo de controle wireless, que irá controlar chaves eletrônicas responsáveis pela conexão dos ramos do espelho de corrente;

- Através do módulo de controle wireless, o usuário poderá se comunicar em rede sem fio com o dispositivo. E então, controlar o fluxo luminoso dos LEDs de forma remota;

- O microcontrolador recebe sua alimentação de um conversor DC-DC, que por sua vez, é alimentado por um barramento de tensão DC proveniente da saída do conversor AC-DC utilizado para alimentar o sistema.

Por ser constituído principalmente por elementos semicondutores, o dispositivo desenvolvido pode ser implementado em circuitos microeletrônicos integrados para o controle de corrente de forma digital.

\subsection{Especificações de comunicação}

Através do módulo de controle wireless, é possível se conectar a uma rede Wi-Fi local e, através do protocolo UDP, receber comandos do usuário por meio de um aplicativo. $\mathrm{O}$ firmware do módulo tem que estar preparado para receber os pacotes, interpretá-los e acionar os GPIOs correspondentes, bem como o aplicativo deve enviar os dados corretamente para o controle de luminosidade.

O User Datagram Protocol mais conhecido pela sigla UDP, é um protocolo que atua na camada de transporte. Juntamente com o Transmission Control Protocol (TCP), que foca na garantia de recebimento dos pacotes de dados e também sua integridade, o UDP é um dos protocolos mais usados na camada citada. (I. Coonjah, 2015). O protocolo UDP se destaca devido sua velocidade de trabalho com o fluxo de dados, mas, em contrapartida, não traz garantias quanto o recebimento e a integridade dos pacotes de dados. Sendo assim, é muito utilizado em aplicações onde há necessidade de uma comunicação em baixa latência, sem necessidade de alta precisão, ao contrário do TCP (A. R. Rind,2006).

O protocolo UDP foi escolhido devido à necessidade de um enlace de forma rápida para que a dimerização fosse realizada em tempo real, mas também, sem que fosse necessário a confirmação do recebimento e integridade dos dados. A figura 4 apresenta o diagrama de comunicação UDP utilizado para conectar o usuário ao dispositivo de controle de iluminação.

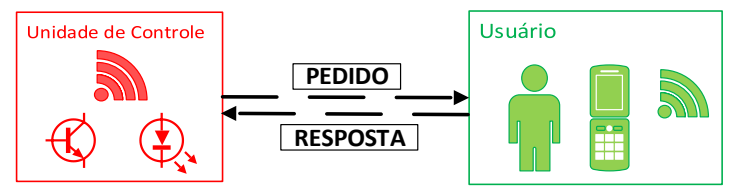

Figura 4 - Arquitetura de implementação do protocolo UDP com o dispositivo. 


\subsection{Módulo de controle wireless: ESP8266}

De acordo com a fabricante Espressif Systems o ESP8266 é um System on Chip (SoC) com grande integração wireless, projetado para plataformas móveis com restrição de espaço e potência. Ele oferece capacidade de incorporar recursos de WiFi dentro de outros sistemas, ou para funcionar como uma aplicação standalone, com o menor custo e com mínimo requisito de espaço. (Espressif, 2018)

O ESP8266EX integra switch de antena, RF Balun, amplificador de potência, amplificador de recepção de baixo ruído, filtros e módulos de gerenciamento de energia. O design compacto minimiza o tamanho da placa de circuito impresso e requer poucos circuitos externos.

Além das funcionalidades de Wi-Fi, o ESP8266EX também integra uma versão aprimorada do processador de 32 bits que opera em frequências ajustáveis de 80 a $160 \mathrm{MHz}$ da Tensilica e SRAM no chip, do inglês Static Random Access Memory. Pode ser conectado com sensores externos e outros dispositivos através dos GPIOs. Esses recursos citados e as especificações do ESP 8266 podem ser observados nas figuras 4 e 5 respectivamente: (Espressif, 2018)

Tabela 1. Especificações do ESP8266

\begin{tabular}{|c|c|}
\hline Componentes & Referências \\
\hline CPU & $\begin{array}{c}\text { Tensilica L106 32-bit } \\
\text { processor }\end{array}$ \\
\hline Wi-Fi & $802.11 \mathrm{~b} / \mathrm{g} \mathrm{n}$ \\
\hline Faixa de Frequência & $2.4 \mathrm{GHz}$ \\
\hline \multirow{3}{*}{ Protocolos de Rede } & IPv4, TCP/UDP, \\
& $\begin{array}{c}\text { HTTP/FTP, 802.11 } \\
\text { b/g/n/, protocolo WLAN } \\
\text { MAC }\end{array}$ \\
\hline Periféricos & UART, SDIO, SPI, I2C, \\
& I2S. \\
\hline Tensão de operação & $2,5 \mathrm{~V} \sim 3,6 \mathrm{~V}$ \\
\hline Tensão de operação & $3.3 \mathrm{~V}$ \\
\hline Corrente de operação & $80 \mathrm{~mA}$ \\
\hline
\end{tabular}

\subsection{Framework para desenvolvimento do aplicativo: $Q T$}

Trata-se de uma multiplataforma baseada em linguagem de programação $\mathrm{C}++$, responsável pelo desenvolvimento de interfaces gráficas. O QT é baseado em módulos, sendo os dois principais o qt essentials e o qt addon. O essentials carrega todas as bibliotecas responsáveis pelo funcionamento do programa e o addon apenas as bibliotecas adicionais que não são alocadas nas distribuições básicas, o que permite com que o produto evolua com o tempo e não se torne ultrapassado através da inserção de novas bibliotecas no módulo (Z. Guigen 2010). O QT também se trata de um programa de alta performance devido a linguagem no qual é baseado, por esse motivo e também por ser um programa de fácil aprendizado, conta com uma comunidade de mais de um milhão de desenvolvedores, o que nos dá acesso à uma comunidade de grandes proporções caso surjam problemas durante o desenvolvimento. Além disso, o framework possui diversas ferramentas de desenvolvimento e API's, que colaboraram para criação de um aplicativo com comunicação web baseado no protocolo UDP, cujo qual foi utilizado para realizar o controle dos níveis de iluminação.

\section{IMPLEMENTAÇÃO}

Conforme apresentado na arquitetura de hardware na figura 3, o protótipo desenvolvido é constituído por três unidades básicas: Um conversor DC-DC, um módulo de controle wireless e um circuito espelho de corrente com múltiplos ramos.

A alimentação do sistema é proveniente de uma fonte AC-DC de bancada, fornecendo uma tensão de $30 \mathrm{~V}$ para o módulo IoT para controle de iluminação. Essa alimentação também será fornecida a um conversor DC-DC responsável por condicionar a tensão a $5 \mathrm{~V}$ para alimentar o módulo de controle wireless.

O módulo de controle Wireless é responsável por tornar o protótipo em um objeto com conectividade. Essa conexão se dará através do ESP8266 nodeMCU que é dotado do padrão Wi-Fi (IEEE 802.11) que atua na faixa de $2.4 \mathrm{GHz}$ para comunicação em rede. Como citado anteriormente, esse módulo utilizará o protocolo UDP para a realização da dimerização da iluminação. Além disso, vale ressaltar que essa implementação está inserida em uma rede de área local ou LAN (Local Access Network), ou seja, os endereços de IP (Internet Protocol) são conhecidos previamente.

Por fim, o elemento a ser controlado no sistema é a corrente no circuito espelho de corrente com cinco ramos. Nesse circuito o número de transistores bipolares por ramo deu-se de maneira crescente, isto é, o primeiro ramo possui apenas um transistor, o segundo ramo possui dois transistores, seguindo assim segue até o quinto ramo, com cinco transistores.

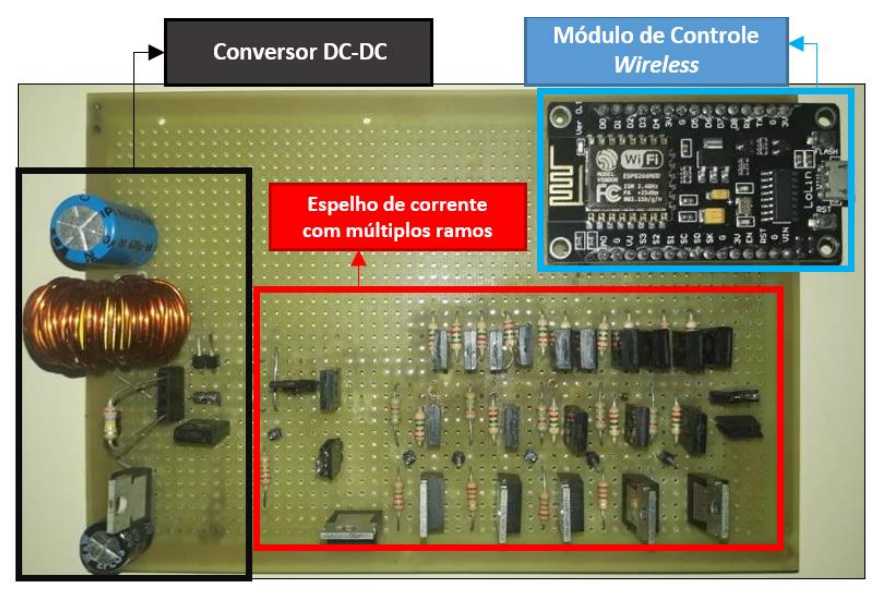

Figura 5 - Protótipo de teste desenvolvido.

Para realizar os testes, foi definido um resistor no valor de $1 \mathrm{k} \Omega$ a fim de regular a corrente no ramo principal, o que permitiu produzir uma corrente de aproximadamente $30 \mathrm{~mA}$ em cada transistor. No entanto, considerando o erro devido a razão de transferência de corrente, sabe-se que a corrente por transistor pode ser reduzida à medida que os ramos do espelho são acionados. 
O protótipo receberá alimentação em corrente contínua de $30 \mathrm{~V}$, que alimentará o bloco de conversão DC-DC e a entrada do espelho de corrente. O conversor DC-DC foi elaborado através de um circuito regulador step-down com o regulador LM2596, que abaixa a tensão de entrada para 5V, necessários para alimentação do nodeMCU.

A figura 6 apresenta a interface do aplicativo elaborado no QT para controle de iluminação. $O$ aplicativo possibilita a regulação do nível de iluminação em uma escala de 0 a 15. Nessa imagem é possível visualizar um exemplo de três níveis diferentes selecionados pelo usuário.

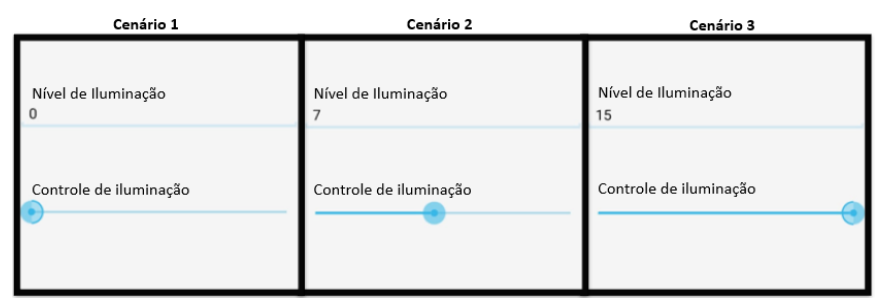

Figura 6 - Interface do aplicativo desenvolvida para realizar o controle de iluminação.

\section{RESULTADOS E DISCUSSÕES}

Na figura 7, é possível visualizar os cenários de teste utilizados para coleta de resultados. Além disso, é apresentada a luminária utilizada sob alguns níveis de controle de iluminação realizados através do dispositivo.

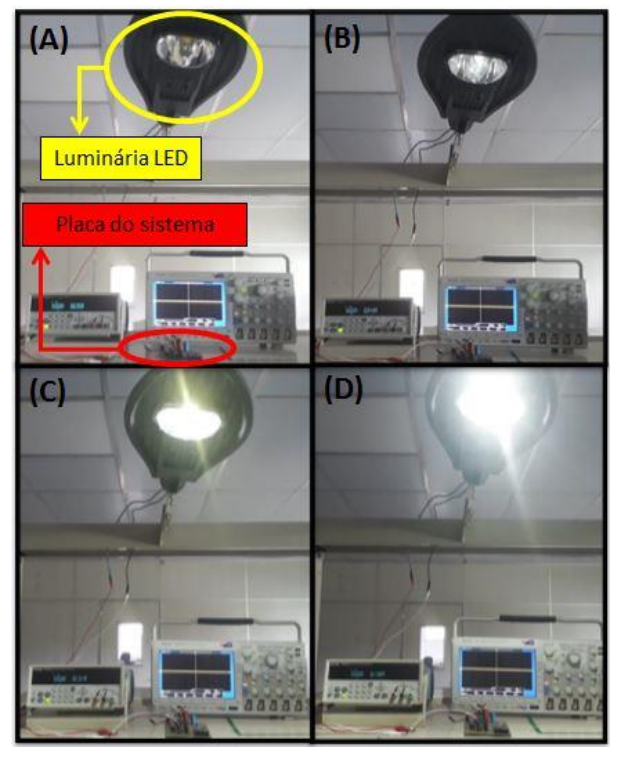

Figura 7 - Ambiente de testes. A: Cenário 1. B: Luminária acesa no primeiro nível de iluminação. C: Cenário 2. D: Cenário 3.

Com 5 ramos de espelho, de acordo com a arquitetura ilustrada na figura 3 , foram obtidos diversos níveis de controle de fluxo luminoso possíveis com o dispositivo. Os níveis de corrente no LED aumentaram a medida em que os GPIOs do ESP8266 nodeMCU comutavam para nível lógico alto. A relação entre a variação de corrente e potência no $\mathrm{CoB}$ e eficiência do módulo, de acordo com o acionamento individual de cada GPIO, é apresentada na tabela 2.

Tabela 2. Variação de corrente e potência no CoB e eficiência do módulo IoT por GPIO de controle acionado

\begin{tabular}{|c|c|c|c|}
\hline $\begin{array}{c}\mathbf{N}^{\circ} \text { do } \\
\text { GPIO }\end{array}$ & $\begin{array}{c}\text { Corrente } \\
(\mathbf{m A})\end{array}$ & $\begin{array}{c}\text { Potência } \\
(\mathbf{W})\end{array}$ & $\begin{array}{c}\text { Eficiência } \\
(\mathbf{\%})\end{array}$ \\
\hline 0 & 30 & 0,82 & 42 \\
\hline 1 & 59,2 & 1,63 & 58 \\
\hline 2 & 88,5 & 2,45 & 72 \\
\hline 3 & 114 & 3,20 & 74 \\
\hline 4 & 142 & 4,02 & 75 \\
\hline
\end{tabular}

Visto que os transistores operam em região linear, quando compõem a estrutura de um espelho de corrente, optou-se por não utilizar a capacidade total do $\mathrm{CoB}$ para evitar o aquecimento destes componentes. A eficiência deste sistema, conforme o apresentado na tabela 2 , foi obtida através da razão entre a potência consumida pelo CoB de LEDs e a potência de entrada do módulo IoT.

Como previsto, com o acréscimo de transistores conectados, o incremento da corrente a cada acionamento foi reduzido. Isso pode ser visualizado na figura 8 quando ocorre a conexão dos GPIOs 3 e 4, onde há redução na inclinação da curva de crescimento da corrente nos LEDs. Sabendo que a tipologia de circuitos reguladores por espelhos de corrente trabalha em regiões lineares de condução, os elementos semicondutores podem vir a dissipar energia na forma de calor, reduzindo a eficiência do circuito. Devido os primeiros acionamentos permitirem a condução de correntes pequenas sob o $\mathrm{CoB}$, a diferença de potencial entre os coletores e emissores em paralelo tendem a ser maior nos menores níveis de luminosidade. Portanto esses semicondutores podem dissipar quantidades consideráveis de energia de forma proporcional a corrente circulante e a diferença de potencial entre coletores e emissores.

\section{CONCLUSÕES}

O desenvolvimento do protótipo apresentado nesse artigo, obteve resultados condizentes com o pretendido. Um desses resultados diz respeito à possibilidade de tornar uma luminária LED convencional, que não dispõe de conectividade com a rede de computadores nem de possibilidade de controle, em uma luminária LED inteligente que consiste em um objeto capaz de oferecer serviços de controle remoto através da internet, bem como controle da intensidade luminosa desejada pelo usuário.

O controle da intensidade luminosa se tornou possível, haja vista topologia adotada para o espelho de corrente. Esse espelho de corrente utilizou de conceitos de circuitos transistorizados para o controle de corrente em cada LED, ensejando através disso o controle da iluminação. Essa topologia adotada mostra-se como inovação para aplicações de controle de iluminação, podendo ser adotada em outros sistemas que requerem o controle de corrente. 
Nos próximos trabalhos será possível a redução do tamanho do protótipo, pois os circuitos a transistores podem ser compactados a tamanhos mínimos, em decorrência do avanço tecnológico da área de semicondutores

\section{AGRADECIMENTOS}

Gratidão à Universidade do Estado do Amazonas (UEA) por todo suporte, em especial ao Instituto de Pesquisa vinculado a UEA, HUB: Tecnologia e Inovação, por oferecer todo o aparato técnico para o desenvolvimento do protótipo e realização dos testes. Grato aos amigos Samuel Bruno e Ítalo Tony pelo apoio prestado.

\section{REFERÊNCIAS}

A. A.-Fuqaha et al., "Internet of Things: A Survey on Enabling Technologies, Protocols, and Applications," IEEE Commun.Surveys \& Tutorials, vol. 17 , no. $4,4^{\text {th }}$ qtr. 2015 , pp. 2347-76.

A. R. Rind, K. Shahzad and M. A. Qadir, "Evaluation and comparison of TCP and UDP over Wired-cum-Wireless LAN," 2006 IEEE International Multitopic Conference, Islamabad, 2006, pp. 337-342.

Ala Al-Fuqaha, Mohsen Guizani, Mehdi Mohammadi, Mohammed Aledhari, (2015), "Internet of Things: A Survey on Enabling Technologies, Protocols and Applications", IEEE Communications Surveys \& Tutorials, vol.3, no.5.

C. Perera et al., "A Survey on Internet of Things: From Industrial Market Perspective," IEEE Access, vol. 2, Dec. 2014, pp. 1660-79.

\section{Espressif 2018 - ESP 8266 Datasheet}

I. Coonjah, P. C. Catherine and K. M. S. Soyjaudah, "Experimental performance comparison between TCP vs UDP tunnel using OpenVPN," 2015 International Conference on Computing, Communication and Security (ICCCS), Pamplemousses, 2015, pp. 1-5.

J.ChandraMohan, R.Nagarajan, K.Satheeshkumar, N.AjithKumar, P.A.Gopinath, S.RanjithKumar (2017) "Intelligent Smart Home Automation and Security System using Arduino and Wi-Fi" , International Journal of Engineering and Computer Science, vol 6,issue 3,pp.20694-20698.

J. Kim and S. Park, "12.8 Synchronized floating current mirror for maximum LED utilization in multiple-string linear LED drivers," 2016 IEEE International Solid-State Circuits Conference (ISSCC), San Francisco, CA, 2016, pp. 232-233.

SEDRA, S.; SMITH, K. Microeletrônica. $4^{\text {a }}$. Edição, Pearson Makron Books, São Paulo, Brasil, 2005.

Z. Gui-gen, W. Shuang, C. Lei and C. FuZhou, "The Implementation of oSIP Stack in Developing Qt Software in Embedded Linux," 2010 First International Conference on
Networking and Distributed Computing, Hangzhou, 2010, pp. 137-141.

Ben Pronk and Stefan Verbrugh, Challenges of the Integration of Lighting Systems and Components in IoT, 2018, pp. 50-76. 